\title{
Aneurysmal and obstructive lesions of the left ventricular outflow: evaluation on multidetector computed tomography angiography
}

\author{
Mumun Sinha ${ }^{A, B, D, E, F}$, Niraj N. Pandey ${ }^{A, B, D, E, F}$, Arun Sharma ${ }^{A, B, D, E, F}$, Nitin Parashar ${ }^{A, B, D, E, F}$, Sanjeev Kumar ${ }^{A, B, D, E, F}$, \\ Gautam Sharma ${ }^{A, B, D, E, F}$
}

All India Institute of Medical Sciences, New Delhi, India

\begin{abstract}
The left ventricular outflow is an anatomically complex region situated between the anterior leaflet of the mitral valve and the left ventricular aspect of the muscular and membranous interventricular septum. It gives rise to the aorta, provides support to the aortic valvular cusps, and houses important components of the conduction system. The left ventricular outflow handles high pressures and pressure variations and is subsequently affected by a variety of aetiopathological conditions. Diseases involving the left ventricular outflow can be intraluminal, mural, or extramural, and the consequent complications of the lesions can be local, loco-regional, or even systemic. Appropriate evaluation requires comprehensive multimodality imaging with each modality contributing to assessment of different aspects of diagnosis, lesion characterization, local extension, prognostication for systemic complications and mortality, and the decision for the approach and type of intervention and aggressive follow-up in case non-interventional management is decided. In this review, we briefly describe the relevant anatomy and the gamut of structural abnormalities pertaining to the left ventricular outflow on multidetector computed tomography angiography.
\end{abstract}

Key words: left ventricular outflow, mitral aortic intervalvular fibrosa aneurysm, mitral valve prolapse, aneurysm of membranous interventricular septum.

\section{Introduction}

The left ventricular outflow (LVO) is an anatomically complex region of the left ventricle (LV), which gives rise to the aorta, provides support to the aortic valvular cusps, and houses important components of the conduction system. The region handles tremendous pressures and pressure variations and is affected by a myriad of aetiopathological conditions, some of which are innocuous while others may be symptomatic, progressive, and lethal. The diseases involving the region can be intraluminal (involving its lumen), mural (involving its boundaries), or extramural (pathologies of surrounding structures). Similarly, complications of the lesions can be local, locoregional (from compression of surrounding structures), or even systemic. For the purposes of a comprehensive review, the structural abnormalities of the left ventricular outflow have been broadly divided into aneurysmal and obstructive lesions.

\section{Relevant anatomy}

The LVO is the region of the LV that is situated between the anterior leaflet of the mitral valve (MV) and the left ventricular smooth side of the muscular and membranous interventricular septum (IVS) (Figure 1). Because the IVS is angled at 45 degrees with respect to the median plane, the anterior wall of the LVO becomes the anteromedial wall and the posterior wall becomes the posterolateral wall [1].

The anterior leaflet of the MV (posterolateral wall) is thus displaced from the IVS by the LVO. This leaflet is the larger of the 2 mitral leaflets and is attached to onethird of the annular circumference. Because of its fibrous

Correspondence address:

Dr. Arun Sharma, 148, The Foothills, New Chandigarh (Pb), India, e-mail: drarungautam@gmail.com

Authors' contribution:

A Study design · B Data collection · C Statistical analysis · D Data interpretation · E Manuscript preparation · F Literature search · G Funds collection 


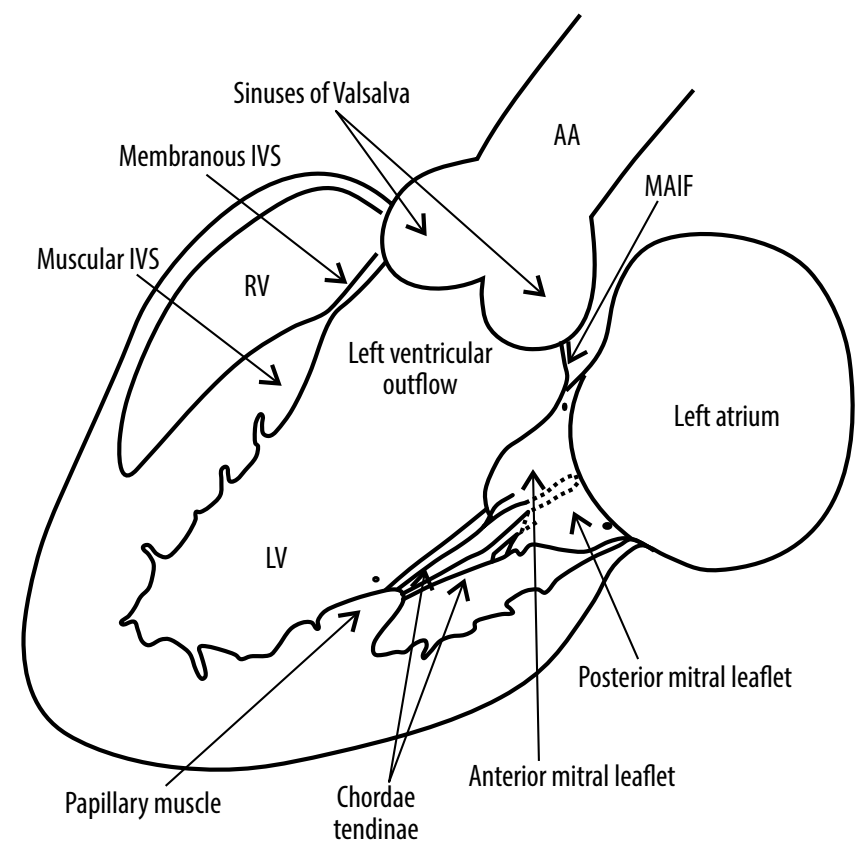

Figure 1. Schematic diagram showing the anatomy of the left ventricular outflow. LV - left ventricle, RV - right ventricle, MAIF - mitral-aortic intervalvular fibrosa, AA - ascending aorta, IVS - interventricular septum

continuity with the aortic valve, this leaflet is also referred to as the aortic leaflet. The region of the fibrous continuity is referred to as the mitral aortic intervalvular fibrosa (MAIF) [1]. The membranous septum is a small compact fibrous part of the IVS that lies between the non-coronary and right coronary aortic cusp and the septal leaflet of the tricuspid valve (TV) and therefore, at its upper end, is continuous with the right lateral wall of the aortic root. Also, the membranous septum is partly atrio-ventricular

Table 1. Classification of structural abnormalities of the left ventricular outflow

\begin{tabular}{|l|l|}
\hline A & Aneurysmal lesions \\
\hline 1 & Aneurysm of the membranous part of the interventricular septum \\
\hline 2 & Prolapse of the anterior leaflet of the mitral valve \\
\hline 3 & Aneurysm of the anterior leaflet of the mitral valve \\
\hline 4 & Aneurysm of the mitral aortic intervalvular fibrosa \\
\hline 5 & Unsupported sinus and aortic cusp prolapse \\
\hline 6 & $\begin{array}{l}\text { Contained aortic root rupture/left ventricular outflow } \\
\text { pseudoaneurysm }\end{array}$ \\
\hline B & Obstructive lesions \\
\hline 1 & Hypertrophic obstructive cardiomyopathy \\
\hline 2 & Systolic anterior motion of the mitral valve \\
\hline 3 & Subaortic stenosis \\
\hline 4 & Shone complex and hypoplastic left heart syndrome \\
\hline 5 & Diverticulum of the anterior mitral leaflet \\
\hline 6 & $\begin{array}{l}\text { Sinus of Valsalva aneurysm burrowing into the interventricular } \\
\text { septum }\end{array}$ \\
\hline 7 & $\begin{array}{l}\text { Post surgical repair or spontaneous closure of perimembranous } \\
\text { ventricular septal defect }\end{array}$ \\
\hline
\end{tabular}

and anatomically separates a small portion of the right atrium nearest the septal leaflet of the TV from the LV. At the junction of the muscular and membranous IVS lies the atrioventricular (AV) bundle [2].

The LVO, being exposed to blood at high systemic pressure, cannot withstand structural weakness caused by genetic or acquired causes (iatrogenic, traumatic, infectious, inflammatory, etc.) and hence requires early attention and intervention.

\section{Structural abnormalities of the left ventricular outflow}

The structural abnormalities of the left ventricular outflow can be broadly divided into aneurysmal and obstructive lesions (Table 1).

\section{Aneurysmal lesions}

\section{Aneurysm of the membranous part of the interventricular septum}

The membranous IVS is in close proximity to the proximalmost great vessel, and being amuscular makes it susceptible to aneurysm formation. The likely aetiologies include idiopathic, trauma, iatrogenic (post trans-aortic myotomy), ischaemic, infectious, and secondary to spontaneous partial or complete closure of a ventricular septal defect (VSD) after 2 years of age [3-5]. It is seen in association with congenital heart disease (CHD) in $0.3 \%$ of cases, with the 2 most common associations being VSD and congenitally corrected transposition of great vessels [6-8].

The outpouching of the membranous septum to the right ventricle (RV) side is most evident during systole with 

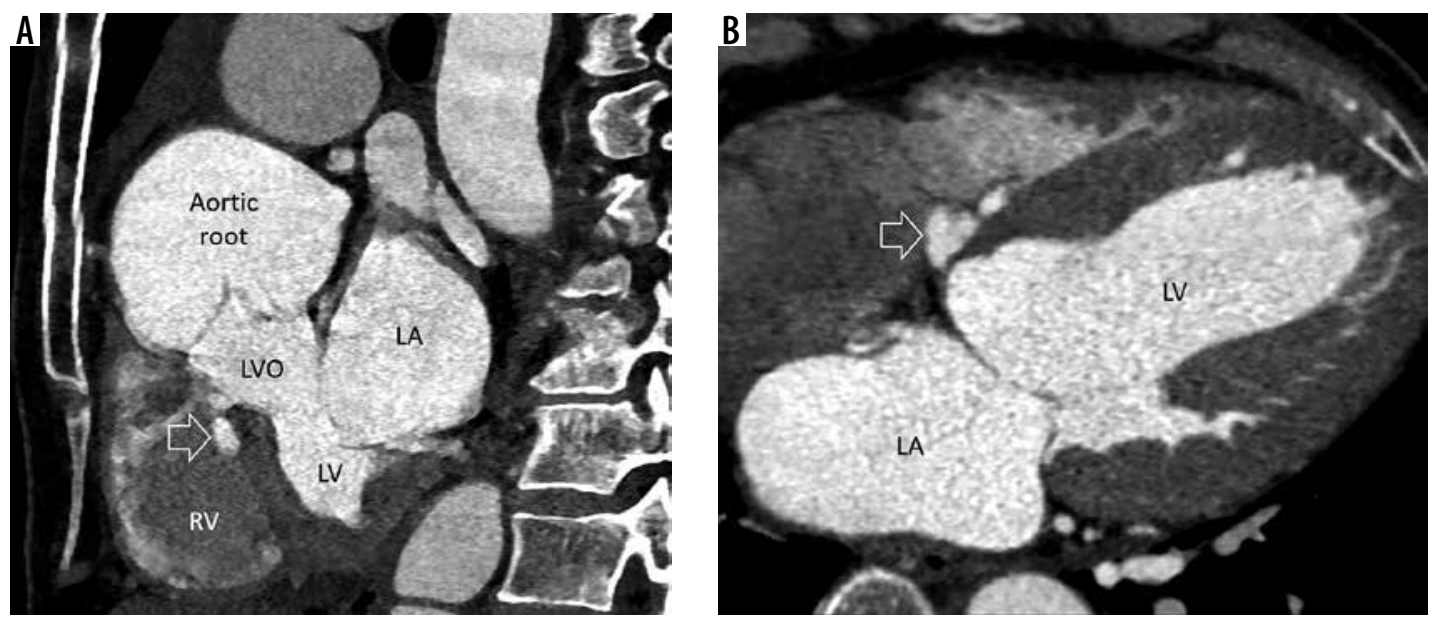

Figure 2. Multiplanar reconstructed image in the short axis (A) and 4-chamber view (B) demonstrate a small, lobulated outpouching (white arrow) from the membranous part of the interventricular septum projecting into the base of right ventricle (RV). LA - left atrium, LV - left ventricle, LVO - left ventricular outflow
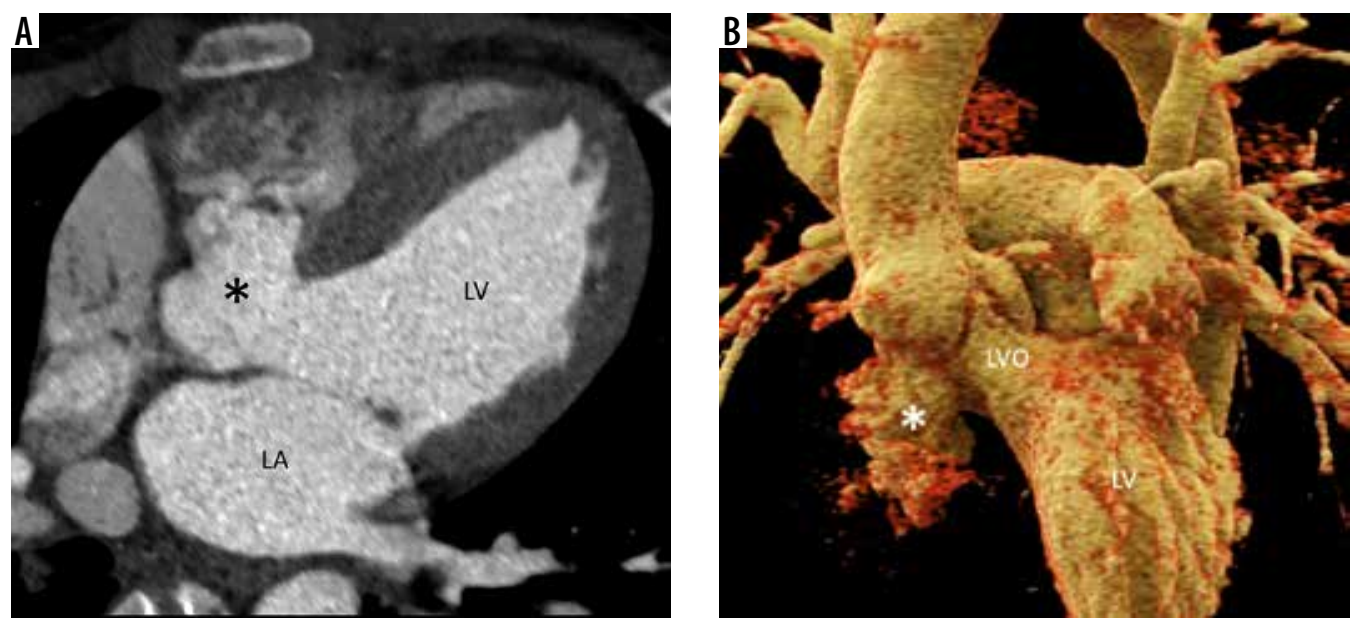

Figure 3. Multiplanar reconstructed image in the 4-chamber view $(A)$ and volume-rendered image $(B)$ demonstrate a lobulated outpouching $\left({ }^{*}\right)$ of the membranous portion of the interventricular septum extending into the right ventricle. LA - left atrium, LV - left ventricle, LV0 - left ventricular outflow

protrusion into the right ventricular outflow tract (RVOT), and it can become imperceptible during diastole. This can be seen both on echocardiography and retrospectively on ECG-gated cardiac computed tomography angiography (CTA) (Figures 2 and 3) [9]. The lesion is often asymptomatic; however, when symptomatic, it is usually secondary to severe RVOT obstruction and dysfunction. Patients are also predisposed to arrhythmias (because of its close relation to the AV bundle), which may disappear after aneurysm repair or may progress to AV blocks after aneurysm resection $[10,11]$. Uncommonly, the aneurysm can be a source of cerebral embolism. Acquired aortic valve incompetence may result from impingement of the aneurysm on the aortic valve leaflets or from prolapse of the aortic leaflets into the aneurysm [12]. Tricuspid regurgitation or left-ventricularto-right atrial shunt may also result [13].

\section{Prolapse of the anterior leaflet of the mitral valve}

This is the most common valvular abnormality in patients with Marfan's syndrome, seen in about $25-75 \%$ of the cases [14].
It is also the most common valve abnormality in the general population, affecting $2-3 \%$ of people, and the most common cause of mitral valve (MV) regurgitation in athletes [15].

Classic mitral valve prolapse (MVP) is defined as superior/atrial displacement of $>2 \mathrm{~mm}$ of the mitral leaflets during systole and a leaflet thickness of at least $5 \mathrm{~mm}$ during diastole (Figure 4) [15]. Non-classic MVP is defined as displacement of $>2 \mathrm{~mm}$ and thickness of $<5 \mathrm{~mm}$ [15]. The incidence of moderate-to-severe MV regurgitation and infective endocarditis (IE) is significantly higher in classic vs. non-classic MVP [15]. Mild billowing with normally appearing valves is considered a normal variant. It can also be seen in association with Wolf-Parkinson-White syndrome and hypertrophic cardiomyopathy (HCM) [16,17].

In patients with MVP without MV regurgitation, the rate of sudden cardiac death (SCD) is no more common than among the general population [15]. Exercise-induced mitral regurgitation may be a predictor of events in patients with MVP without MV regurgitation at rest. However, the incidence of complications with MVP is well studied, the natural history of MVP remains incompletely 

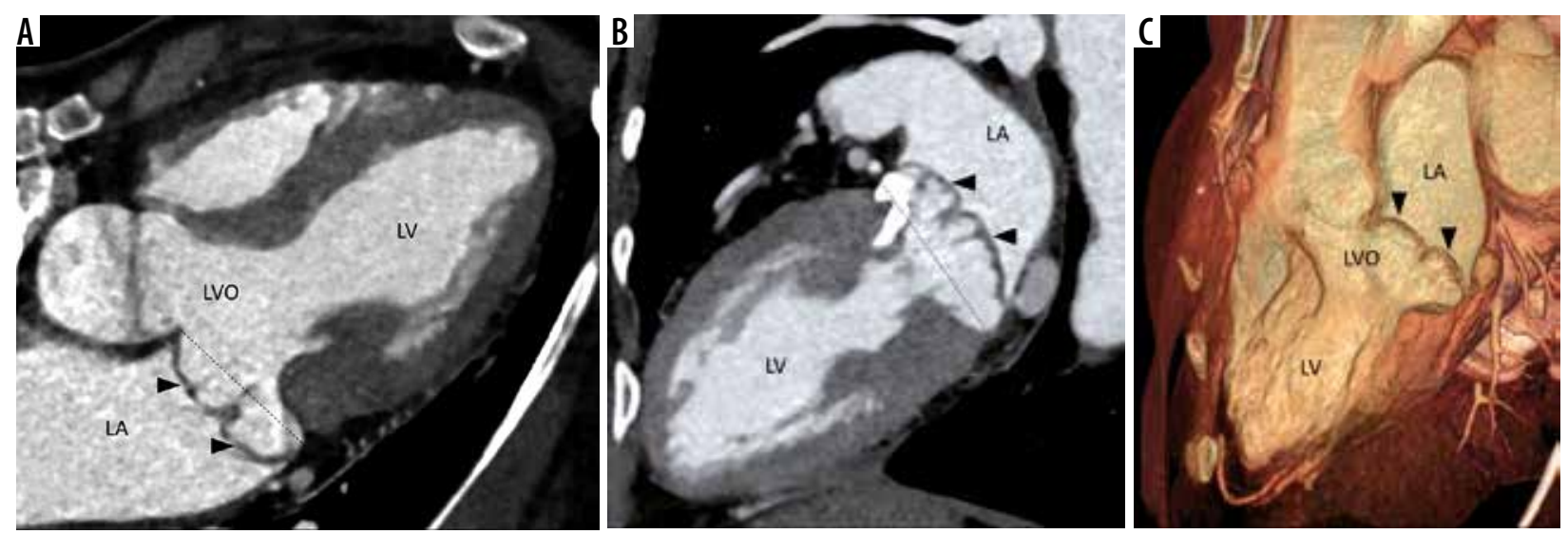

Figure 4. Multiplanar reconstructed image in the 4-chamber view (A), 2-chamber view (B), and volume-rendered image (C) demonstrate prolapse of both the anterior and posterior leaflets of the mitral valve (arrowheads) with resultant widening of the left ventricular outflow (LV0). Note is also made of calcification of the mitral valve annulus. LA - left atrium, LV - left ventricle

elucidated, which necessitates close follow-up to predict progression and complications like atrial and ventricular arrhythmias, congestive heart failure (CHF), severe MV regurgitation, chordal rupture, and IE. Mitral valve repair (MVR) provides long-term survival and life expectancy similar to the general population. Treatment options include small triangular resection (for limited prolapse), chordal transfer of secondary chordae to free margin, and artificial chordoplasty.

\section{Aneurysm of the anterior leaflet of the mitral valve}

This is an uncommon condition that occurs as a complication of IE of the aortic or mitral valve. Other causes are rheumatic heart disease and degenerative disorders of connective tissue (Marfan's, Ehlers Danlos, pseudoxanthoma elasticum, severe non-infectious aortic regurgitation [AR], and MVP) $[18,19]$. The mechanism of development is the jet of AR striking the ventricular surface of the anterior mitral leaflet, causing both physical trauma and occult infection. A bulky aortic vegetation (exceeding 6 $\mathrm{mm}$ in size) can prolapse into the LVO and come in direct contact with the anterior mitral leaflet. There is resultant inflammation and scar, which causes structural weakening of the MV and the formation of outpouching.

On echocardiography and gated cardiac CTA, a persistent bulge of the anterior mitral leaflet is seen protruding towards to left atrium with systolic expansion and diastolic collapse. The differential diagnosis includes MVP, myxomatous MV degeneration, and flail MV, all of which show systolic bulging and diastolic straightening instead of a persistent bulge. Papillary fibroelastoma, cystic myxoma of the $\mathrm{MV}$, and MV blood cysts are other differentials to be considered. Rupture/perforation is the most feared complication leading to acute severe MV regurgitation and CHF. Other complications include endocarditis and thromboembolism [20]. A conservative approach can be followed for small, uncomplicated aneurysms with close follow-up. Large unruptured aneurysms and those with perforation with or with- out significant MV regurgitation need surgical intervention. Because the condition may be missed at surgery for IE when only the ventricular side is inspected, mitral valve aneurysm should be suspected and ruled out on preoperative imaging in all patients with left-sided endocarditis.

\section{Aneurysm of the mitral aortic intervalvular fibrosa}

MAIF is the region of fibrous avascular continuity between the anterior mitral leaflet and the non-coronary aortic valve cusp. Being avascular and fibrotic makes it prone to injury and infection. Outpouching of this region is a pseudoaneurysm communicating with the LVOT. It arises as a complication of aortic valve endocarditis infecting the MAIF with the formation of an abscess cavity which spontaneously ruptures or is surgically drained forming a pseudoaneurysm. Other aetiologies include aortic or mitral valve surgery, chest trauma, and mitral valve endocarditis. Bicuspid aortopathy is also a predisposing condition because and fr it causes congenital weakness of the MAIF [21-23]. On echocardiography, Doppler demonstrates to-and-fro flow between the cavity and LVO. Flow from the LVO into the cavity occurs during systole, and reverse flow occurs during diastole [21]. Fistula into the left atrium creates an eccentric supramitral jet (like MV regurgitation), and therefore MAIF pseudoaneurysm should always be suspected in cases of aortic valve endocarditis and eccentric supramitral jets [24]. When there is a fistula to the aorta, systolic flow occurs towards the pseudoaneurysm and aorta, and diastolic flow occurs from the pseudoaneurysm to the aorta and then to the LVO [21]. Complications other than rupture into adjacent cavity include rupture into the pericardium with or without tamponade, coronary (especially left circumflex artery) compression and myocardial ischaemia from enlarging pseudoaneurysm, pulmonary artery compression causing pulmonary artery hypertension, thromboembolism, and CHF from regurgitant lesions [25]. 

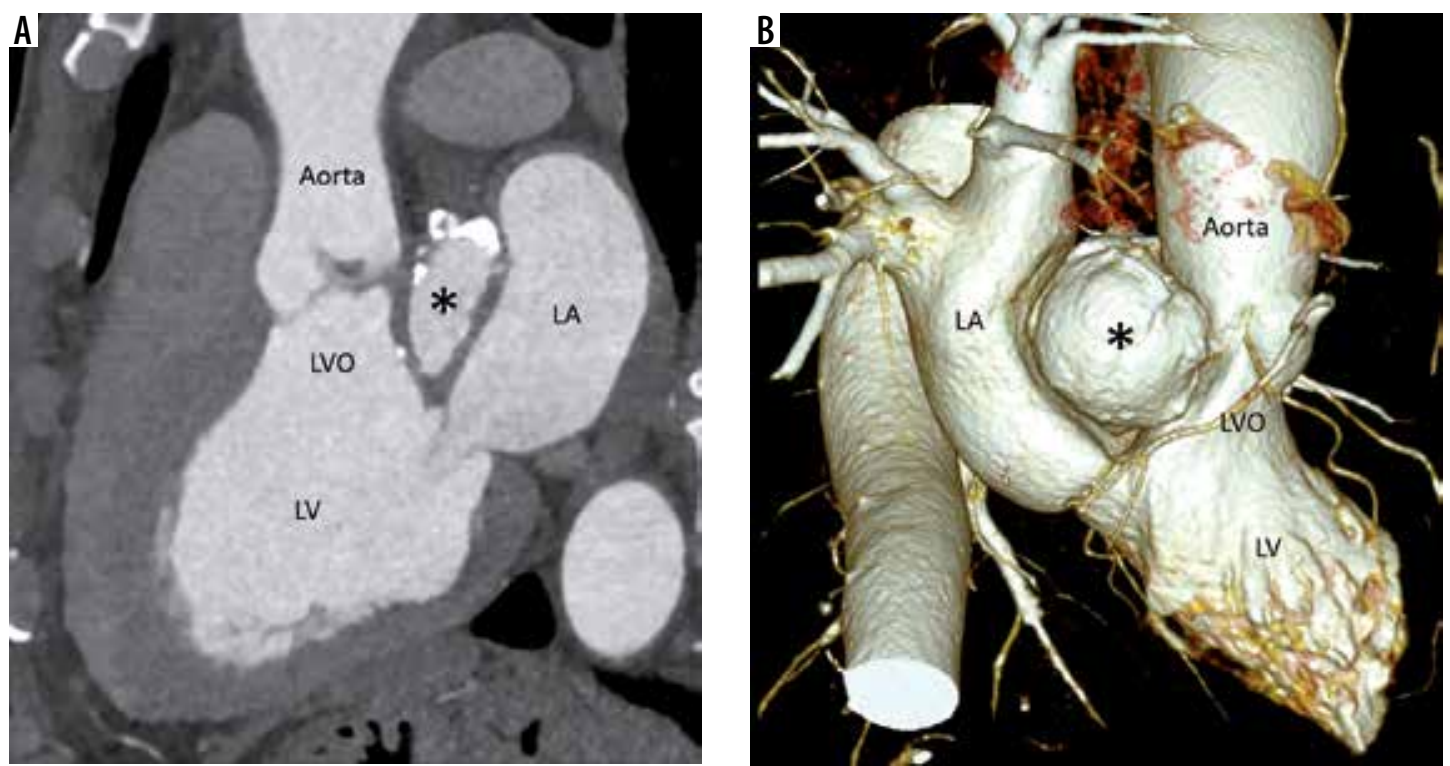

Figure 5. Multiplanar reconstructed image in the oblique sagittal plane (A) and volume-rendered image (B) show a well-defined mitral-aortic intervalvular fibrosa aneurysm $\left({ }^{*}\right)$ with few peripheral specks of calcification. LA - left atrium, LV - left ventricle, LV0 - left ventricular outflow

The description of local extent, compression, and fistula formation to adjacent structures is best demonstrated on cardiac CTA (Figure 5). Cardiac magnetic resonance imaging is best for quantifying regurgitation, assessing myocardial function, and assessing focal discontinuity in the pseudoaneurysm wall and possible fistula formation. Cardiac CTA also rules out close differential diagnoses like paravalvular abscesses, sinus of Valsalva aneurysms, and aortic root ruptures (contained or not contained). Echocardiography is not always helpful and may even lead to a false diagnosis such as cor triatriatum.

Intervention is recommended even in asymptomatic cases because of the high risk and fatality of complications. High-risk features include size $>3 \mathrm{~cm}$, bicuspid aortic valve, AR, fistula to left atrium or aorta, presence of thrombus, and associated compression of adjacent structures. Waitful watching with imaging follow-up has been argued by some authors, especially in smaller aneurysms $(<3 \mathrm{~cm})$, in patients with high surgical risk. Resection and repair of the lesion with or without aortic valve replacement and homograft replacement of the aortic root is the preferred surgery. Percutaneous device closure can be an option for suitable cases.

\section{Unsupported sinus and aortic cusp prolapse}

In infundibular/outlet VSD, the supporting tissue beneath the aortic sinuses is deficient. Blood flow across a restrictive VSD from the LV to the right ventricle can lead to prolapse of the aortic sinus into the RVOT.

Aortic valve prolapse is defined as downward displacement of cusps below the line of coaptation of the aortic valve leaflets; it is seen in various cardiac disorders and is important in producing regurgitation (pre-existing and treatment induced) in bicuspid aortic valves and ascend- ing aortic diseases [26]. Aortic cusp prolapse is known to be associated with subaortic VSD and prolapse, which can also lead to AR identified by an eccentric jet. Pre-existing or treatment-induced cusp prolapse is an important cause of failure of aortic valve sparing procedures; thus, their identification and correction via plication and resuspension procedures is important for success and improved long-term outcomes [26].

\section{Left ventricular outflow tract/aortic root pseudoaneurysm}

This is a consequence of annular suture dehiscence (due to tight sutures) or prosthetic valve endocarditis (preexisting or new). Endocarditis can itself cause sutural dehiscence in addition to paravalvular leak and periaortic infection. Large pre-surgical aortic diameter is a predisposing factor in non-infective cases $[27,28]$. Because of its non-specific symptoms and poor evaluation using echocardiography, a high index of suspicion is required. Its detection and characterization of location, size, neck, extent, associated thrombosis, haematoma, and vascular compression is best done by cardiac CTA (Figure 6). Complications are life threatening and include rupture, thrombosis, and coronary artery compression; thus, the lesion mandates treatment as soon as it is detected.

\section{Obstructive lesions}

\section{Hypertrophic obstructive cardiomyopathy}

LVO obstruction (defined as gradient $>30 \mathrm{mmHg}$ ) is seen in $70 \%$ of HCM; in half of these cases, it is provoked by physiological or pharmacological means [29]. Asymmetrical septal hypertrophy narrows the LVO and creates a Venturi effect, which pulls the anterior leaflet of the mi- 

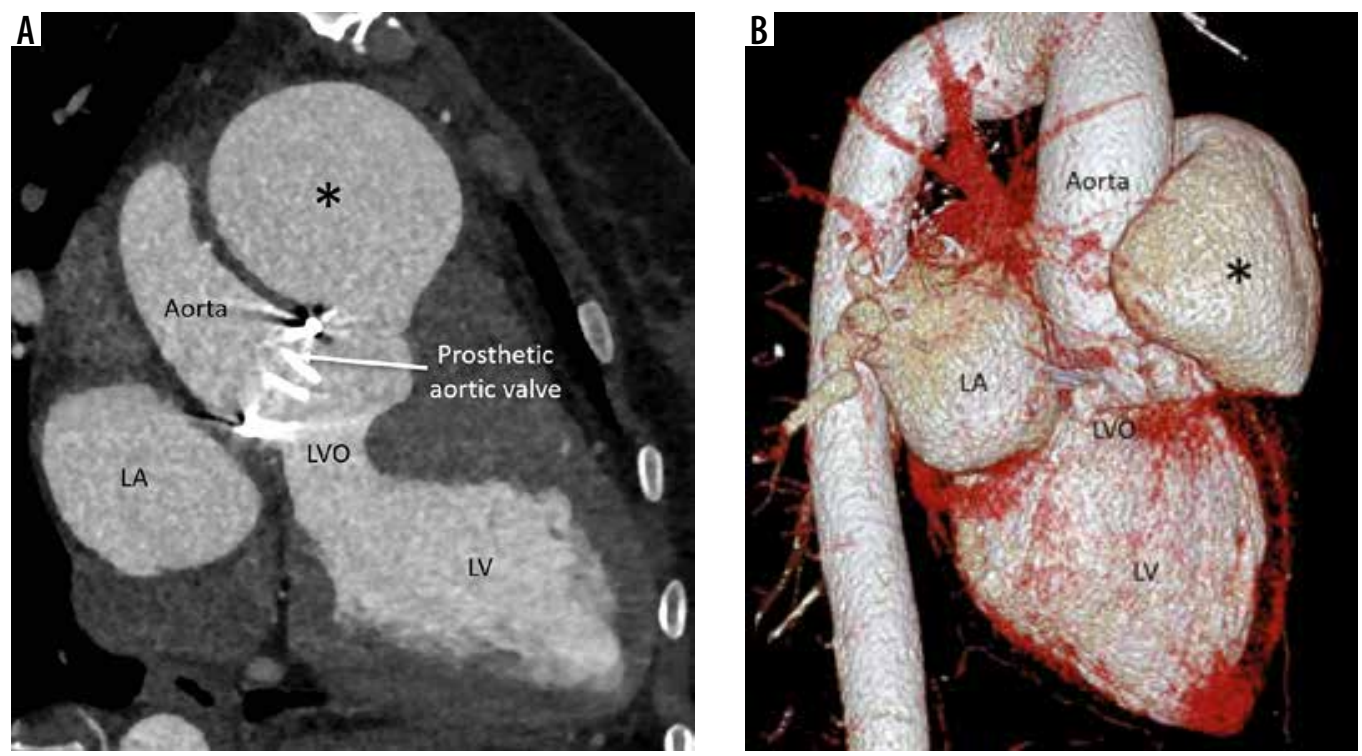

Figure 6. Multiplanar reconstructed image in the oblique sagittal plane (A) and volume-rendered images (B) in a patient after aortic valve replacement show a large pseudoaneurysm $\left(^{*}\right)$ projecting anteriorly from the left ventricular outflow (LVO). LA - left atrium, LV - left ventricle

tral valve during systole. The combination of hypertrophy and systolic anterior motion (SAM) of the anterior mitral leaflet is responsible for dynamic subaortic obstruction and posteriorly directed mitral regurgitation (Figure 7). The obstruction increases the afterload and oxygen demand, causes diastolic dysfunction, and reduces coronary perfusion pressure. SAM leading to LVO obstruction and MV regurgitation is associated with a high ( 20\%) risk of sudden cardiac death (SCD) [30]. Therapies are aimed at reducing the $\mathrm{LVO}$ gradient and include beta and calcium channel blockers, surgical myomectomy, and alcohol septal ablation.

Cardiac magnetic resonance (MR) establishes the baseline (regurgitation and LVO gradients assessed on phase contrast MR imaging) and prognosticates (most importantly can detect and quantitate delayed enhancement). Post intervention assessment and follow-up are other indications of cardiac MR.

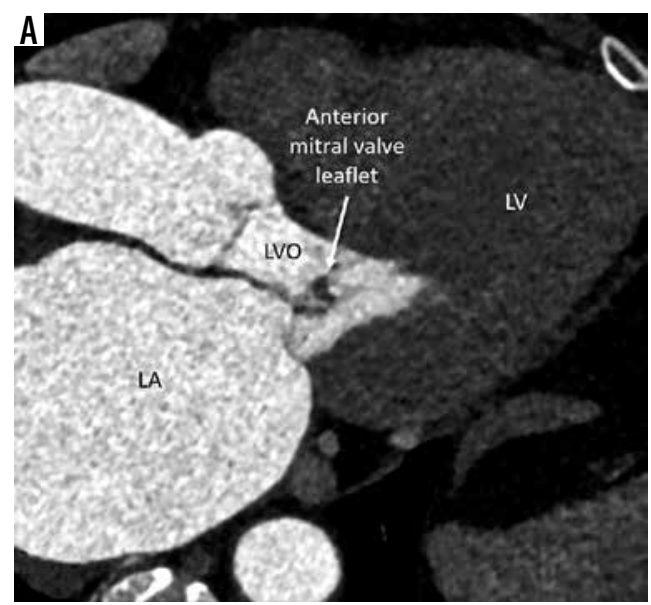

Systolic anterior motion of the mitral leaflet in the absence of HCM

Abnormal systolic anterior motion and LVO obstruction can be the presenting features of Takotsubo cardiomyopathy [31]. SAM is rarely seen in unoperated MV and is probably due to abnormal subvalvular apparatus that alter the position of valve coaptation (anterior and inward). This isolated chordal SAM can occur in the presence (e.g. transposition of great arteries) or absence of any known cardiac abnormality. It can also be a reversible manifestation of cardiac allograft rejection. Other medical causes include diabetes mellitus, myocardial infarction, hypertension, dobutamine stress echocardiography, and general anaesthesia. Patients with rigid mitral repair rings and annular undersizing are predisposed to SAM because of disruption of mitral annular and aortic root dynamics and narrowing of the mitral-aortic angle [32]. SAM can also occur post-

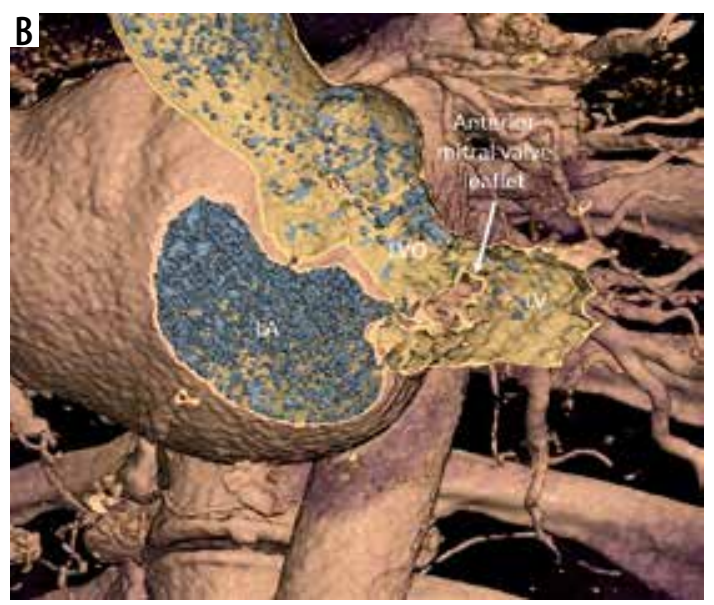

Figure 7. Multiplanar reconstructed image in the oblique axial plane (A) and virtual dissection image (B) in the systolic phase, in a patient with hypertrophic cardiomyopathy, shows thickening of the anterior mitral valve leaflet with anterior motion during systole resulting in left ventricular outflow (LVO) obstruction. LA - left atrium, LV - left ventricle 
aortic valve replacement where pre-existing aortic stenosis pulls the anterior mitral leaflet. Post-surgery afterload reduces and causes the MV to come closer to the LVOT [33].

\section{Subaortic stenosis}

Subaortic stenosis is caused by a variety of lesions, including a thin crescentic subaortic membrane (most common), thick fibromuscular ridge, diffuse narrowing of the LVO, mal-aligned septum, and mitral valve diverticulum, all causing increased left ventricular afterload and hypertrophy (Figure 8).

The stenosis is progressive in children due to increased septal shear stress, which increases cellular proliferation. However, progression is slow in adults (except females and patients more than 30 years of age), especially in discrete stenosis, due to membranes with no set guidelines for timing of follow-up. High-velocity jets can also lead to aortic valvular damage and regurgitation, which increases the cardiac output and transaortic flow, elevating the mean gradients. A direct relation is seen between the lesion severity and AR (including post-operative) with early surgery preventing AR [34]. Subaortic stenosis leads to asymmetric septal hypertrophy and secondary dynamic LVO obstruction; thus, differentiating it from primary obstructive HCM is challenging, especially because the obstruction can mask the underlying membrane on transthoracic echocardiography and cardiac MRI where the dephasing jet can obscure the membrane.

Transoesophageal echocardiography and cardiac CTA can complement transthoracic echocardiography in such cases, and in addition they can help to assess the coronary artery. The decision for treatment in equivocal cases is taken using stress testing to look for arrhythmias, increasing gradient, and ischaemia.

\section{Hypoplastic left heart syndrome (HLHS) and Shone's complex}

HLHS is a broad spectrum encompassing varying degrees of hypoplasia or atresia of left-sided heart valves, left ven- tricle, and ascending aorta. It is a lethal defect which invariably manifests in the first year of life. Shone's complex in its complete form is a combination of parachute mitral valve (causing restricted valve opening), supravalvar mitral ring, subvalvar aortic stenosis, and coarctation of aorta. The lesion is likely to become symptomatic by the age of 2 years, with features of CHF.

\section{Diverticulum of the anterior mitral valve leaflet}

Diverticulum of the mitral leaflet arises as a complication of IE. Infectious destruction of the valve and fibrosis causes thinning and outpouching of the valve. Non-infectious diverticula, most likely localized myxomatous degeneration, have also been reported [35]. The diverticulum expands during systole due to the pull/suction effect on it. This can also lead to mitral regurgitation, which can be successfully treated by MV replacement. The diverticulum can be attached to the IVS by fibrous cord causing a fixed subaortic stenosis [36].

\section{Sinus of Valsalva aneurysm burrowing into the IVS}

Aneurysms of sinus of Valsalva are rare cardiac anomalies. The aneurysms are mostly congenital and less commonly acquired, secondary to infection, trauma, iatrogenic, autoimmune inflammation, atherosclerotic, etc. Sinus of Valsalva aneurysms are associated with supracristal type of VSD into which the aortic cusp may prolapse, leading to AR. AR may also result from deformation of the aortic leaflets in those without a VSD. The aneurysms are usually asymptomatic, unless they rupture. Unruptured aneurysms $(\sim 34 \%)$ can rarely cause symptoms secondary to coronary compression [37]. Symptoms generally occur when the aneurysm ruptures into a cardiac chamber creating a fistula and CHF. Presence of severe AR should raise the possibility of rupture or communication of the aneurysm (mostly the left sinus) with the LVO or left ventricle. Rupture of a small aneurysm is symptomatic only if CHF develops from long-standing volume overload.
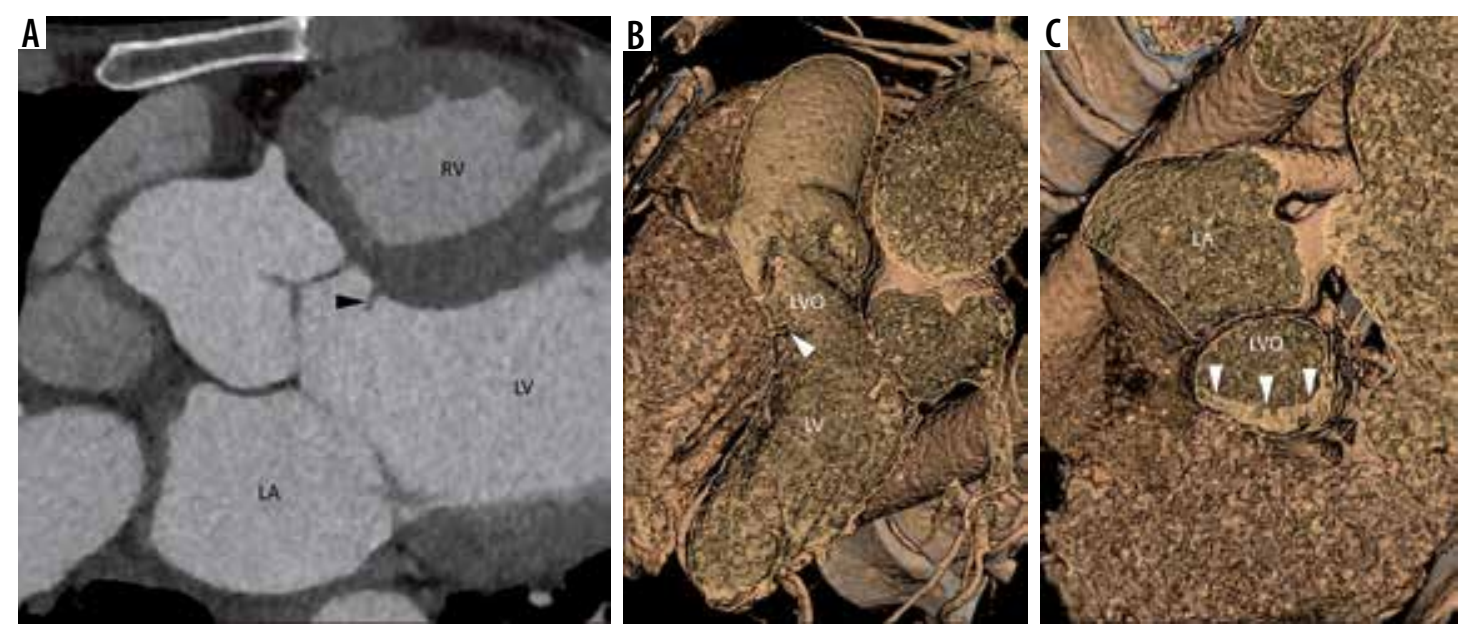

Figure 8. Multiplanar reconstructed image in the oblique axial plane $(A)$ and virtual dissection images $(B, C)$ show a thin eccentric subaortic membrane (arrowhead) causing partial obstruction of the left ventricular outflow. LA - left atrium, LV - left ventricle, RV - right ventricle 

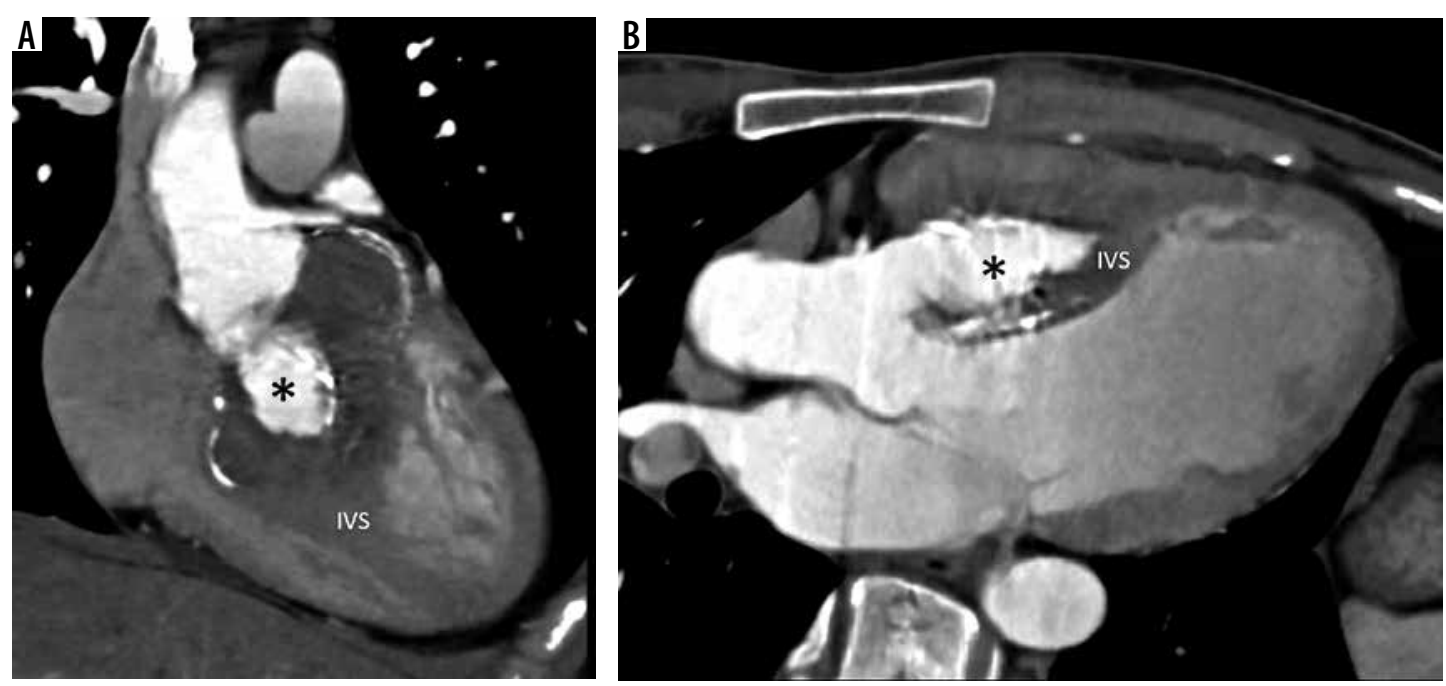

Figure 9. Multiplanar reconstructed image in the oblique coronal plane (A) and oblique axial plane (B) reveals a peripherally thrombosed sinus of Valsalva aneurysm $\left(^{*}\right)$ arising from the right coronary sinus and seen burrowing into the interventricular septum (IVS)

The aneurysm burrowing into the IVS represents a rupture contained by the muscle fibres of the septum (Figure 9). The aneurysm almost always arises from the right coronary sinus and very rarely from the left sinus. Such a dissecting burrowing aneurysm has a high propensity to cause $\mathrm{AV}$ conduction defects and LVO obstruction [38].

\section{Post-surgical repair or spontaneous closure of perimembranous ventricular septal defect}

Few patients may present with varying degrees of LVO obstruction after surgical repair of VSD [39]. While some of these patients may have predisposing congenital LVO malformations, others without such predispositions may also present with membranous subaortic stenosis after surgical repair, presumed to be consequent to the altered flow patterns $[39,40]$. The membranous and fibrous tissue that closes VSD spontaneously may also rarely protrude into the LVO, resulting in stenosis.

\section{Conclusions}

A diverse group of pathological conditions involve the LVO. An aetiology such as infection may affect differ- ent components of the LVO, causing different imaging manifestations. On the other hand, different aetiologies (infection vs. connective tissue disorder) can cause similar imaging manifestations by similar pathological mechanisms of ultimately weakening the region. Appropriate evaluation requires comprehensive multimodality imaging, with each modality contributing to different aspects of diagnosis, lesion characterization, local extension, prognostication for systemic complications and mortality, the decision about the route and type of intervention, and aggressive follow-up if non-interventional management is decided [41]. While CTA is the gold standard for anatomical characterization of local extension, MR is indispensable for haemodynamics, diagnosing focal discontinuities, cardiac function, and fibrosis evaluation. Both these imaging modalities complement each other and cover the loopholes and ambiguity of echocardiographic evaluation.

\section{Conflict of interest}

The authors report no conflict of interest.

\section{References}

1. Walmsley R. Anatomy of left ventricular outflow tract. Br Heart J 1979; 41: 263-267.

2. Saremi F, Hassani C, Sánchez-Quintana D. Septal atrioventricular junction region: comprehensive imaging in adults. Radiographics 2016; 36: 1966-1986.

3. Jain AC, Rosenthal R. Aneurysm of the membranous ventricular septum. Br Heart J 1967; 29: 60-63.

4. Edelstein J, Charms BL. Ventricular septal aneurysms. A report of two cases. Circulation 1965; 32: 981-984.

5. Yilmaz AT, Ozal E, Arslan M, Tatar H, Oztürk OY. Aneurysm of the membranous septum in adult patients with perimembranous ventricular septal defect. Eur J Cardiothorac Surg 1997; 11: 307-311.

6. Choi M, Jung JI, Lee BY, Kim HR. Ventricular septal aneurysms in adults: findings of cardiac CT images and correlation with clinical features. Acta Radiol 2011; 52: 619-623.

7. Yavuz S, Eris C, Goncu T, Sezen M, Ata Y, Turk T. An incidental aneurysm of the interventricular membranous septum. Arch Iran Med 2010; 13: 363-364. 
8. Allwork SP, Bentall HH, Becker AE, et al. Congenitally corrected transposition of the great arteries:morphologic study of 32 cases. Am J Cardiol 1976; 38: 910-923.

9. Carcano C, Kanne JP, Kirsch J. Interventricular membranous septal aneurysm: CT and MR manifestations. Insights Imaging 2016; 7: 111-117.

10. Graffigna A, Minzioni G, Ressia L, Vigano M. Surgical ablation of ventricular tachycardia secondary to congenital ventricular septal aneurysm. Ann Thorac Surg 1994; 57: 921-924.

11. Abdul Jabbar A, Mufti O, Mazur W, et al. Isolated aneurysms of the membranous ventricular septum without residual shunts: systematic review and description of 3 cases in adults. J Ultrasound Med 2017; 36: $869-878$.

12. Sharma M, Elmi F. Interventricular membranous septal aneurysm incidentally diagnosed during computed tomographic angiography in a patient with infrequent supraventricular tachycardia. Clin Pract 2017; 7: 921

13. Tanaka K, Yasunaga H, Egashira A, et al. Aneurysm of the membranous ventricular septum with ventricular septal defect, mitral and tricuspid insufficiency. Jpn J Thorac Cardiovasc Surg 1998; 46: 1009-1013.

14. Delling FN, Vasan RS. Epidemiology and pathophysiology of mitral valve prolapse: new insights into disease progression, genetics, and molecular basis. Circulation 2014; 129: 2158-2170.

15. Freed LA, Levy D, Levine RA, et al. Prevalence and clinical outcome of mitral-valve prolapse. N Engl J Med 1999; 341: 1-7.

16. Drake CE, Hodsden JE, Sridharan MR, Flowers NC. Evaluation of the association of mitral valve prolapse in patients with Wolff-Parkinson-White type ECG and its relationship to the ventricular activation pattern. Am Heart J 1985; 109: 83-87.

17. Petrone RK, Klues HG, Panza JA, Peterson EE, Maron BJ. Coexistence of mitral valve prolapse in a consecutive group of 528 patients with hypertrophic cardiomyopathy assessed with echocardiography. J Am Coll Cardiol 1992; 20: 55-61.

18. Janardhanan R, Kamal MU, Riaz IB, Smith MC. Anterior mitral valve aneurysm: a rare sequelae of aortic valve endocarditis. Echo Res Pract 2016; 3: K7-K13.

19. Işlak Z, Küçük U, Uçak A, Uz O, Doğan M. Ruptured mitral valve aneurysm due to infective endocarditis. Eur Heart J Cardiovasc Imaging 2015; 16: 1030.

20. Reid CL, Chandraratna AN, Harrison E, et al. Mitral valve aneurysm: clinical features, echocardiographic-pathologic correlations. J Am Coll Cardiol 1983; 2: 460-464.

21. Bonou M, Papadimitraki ED, Vaina S, et al. Mitral-aortic intervalvular fibrosa pseudoaneurysm. J Cardiovasc Ultrasound 2015; 23: 257-261.

22. Gajjar T, Jinaga NR, Desai N. Aneurysm of mitral-aortic intervalvular fibrosa - a review. Indian J Thorac Cardiovasc Surg 2017; 33: 142.

23. Takamiya Y, Miura S, Sako H, et al. Pseudoaneurysm of the mitral-aortic intervalvular fibrosa following infective endocarditis in a patient with acute heart failure: a case report. J Cardiol 2007; 49: 353-356.

24. Moorthy N, Kumar S, Tewari S, et al. Mitral-aortic intervalvular fibrosa aneurysm with rupture into left atrium: an uncommon cause of acute dyspnea. Heart Views 2012; 13: 13-15.
25. Parakh N, Yadav N, Kathuria S, et al. Pseudo-aneurysm of mitral aortic intervalvular fibrosa: two case reports. Indian Heart J 2013; 65: 100-103.

26. Boodhwani M, de Kerchove L, Watremez C, et al. Assessment and repair of aortic valve cusp prolapse: implications for valve-sparing procedures. J Thorac Cardiovasc Surg 2011; 141: 917-925.

27. Shariff MA, Martingano D, Khan U, et al. Left ventricular outflow tract pseudoaneurysm after aortic valve replacement. Aorta (Stamford) $2015 ; 3: 167-171$.

28. Tsai IC, Hsieh SR, Chern MS, et al. Pseudoaneurysm in the left ventricular outflow tract after prosthetic aortic valve implantation: evaluation upon multidetector-row computed tomography. Tex Heart Inst J 2009; 36: 428-432.

29. Baxi AJ, Restrepo CS, Vargas D, et al. Hypertrophic cardiomyopathy from a to z: genetics, pathophysiology, imaging, and management. Radiographics 2016; 36: 335-354.

30. Liew AC, Vassiliou VS, Cooper R, Raphael CE. Hypertrophic cardiomyopathy - past, present and future. J Clin Med 2017; 6: 118.

31. Shah BN, Curzen NP. Reversible systolic anterior motion of the mitral valve and left ventricular outflow tract obstruction in association with Takotsubo syndrome. Echocardiography 2011; 28: 921-924.

32. Ibrahim M, Rao C, Ashrafian H, et al. Modern management of systolic anterior motion of the mitral valve. Eur J Cardiothorac Surg 2012; 41: 1260-1270.

33. Luckie M, Khattar RS. Systolic anterior motion of the mitral valve beyond hypertrophic cardiomyopathy. Heart 2008; 94: 1383-1385.

34. Devabhaktuni SR, Chakfeh E, Malik AO, et al. Subvalvular aortic stenosis: a review of current literature. Clin Cardiol 2018; 41: 131-136.

35. Jaladi R, Levy WK, Addonizio PV. Diverticulum arising from mitral valve. J Am Soc Echocardiogr 2004; 17: 288-289.

36. Agathos EA, Moran M, Mangion J, et al. "Diverticula" of anterior mitral valve leaflet as a cause of subvalvular aortic stenosis. J Heart Valve Dis 1996; 5: 309-311.

37. Bricker AO, Avutu B, Mohammed TL, et al. Valsalva sinus aneurysms: findings at CT and MR imaging. Radiographics 2010; 30: 99-110.

38. Park KH, Shin DG, Son CW, et al. Aneurysm of sinus of valsalva dissecting into the interventricular septum after aortic valve replacement: diagnosis by echocardiography and magnetic resonance imaging and treatment with surgical sealant. Korean Circ J 2011; 41: 464-468.

39. Gabriels C, De Backer J, Pasquet A, et al. Long-term outcome of patients with perimembranous ventricular septal defect: results from the belgian registry on adult congenital heart disease. Cardiology 2017; 136: 147-155.

40. Cape EG, Vanauker MD, Sigfússon G, et al. Potential role of mechanical stress in the etiology of pediatric heart disease: septal shear stress in subaortic stenosis. J Am Coll Cardiol 1997; 30: 247-254

41. Hamatani Y, Shiraishi I, Nishii T, et al. Multimodality imaging and three-dimensional printed model in patients with left ventricular outflow tract obstruction. ESC Heart Fail 2020; 7: 320-324. 